\title{
Induced resonance makes light sterile neutrino dark matter cool
}

\author{
F. Bezrukov ${ }^{*}$ \\ The University of Manchester, School of Physics and Astronomy, \\ Oxford Road, Manchester M13 9PL, United Kingdom \\ A. Chudaykin ${ }^{\dagger}$ and D. Gorbunov \\ Institute for Nuclear Research of the Russian Academy of Sciences, \\ 60th October Anniversary prospect 7a, Moscow 117312, Russia \\ and Moscow Institute of Physics and Technology, Institutsky pereulok 9, Dolgoprudny 141700, Russia
}

(Received 1 October 2018; revised manuscript received 11 December 2018; published 9 April 2019)

\begin{abstract}
We describe two new generation mechanisms for dark matter (DM) composed of sterile neutrinos with $\mathcal{O}(1) \mathrm{keV}$ mass. The model contains a light scalar field that coherently oscillates in the early Universe and modulates the Majorana mass of the sterile neutrino. In a region of model parameter space, the oscillations between active and sterile neutrinos are resonantly enhanced. This mechanism allows us to produce sterile neutrino DM with a small mixing angle with active neutrinos, thus evading the x-ray constraints. At the same time, the spectrum of produced DM is much cooler than in the case of ordinary oscillations in plasma, opening a window of lower mass DM, which is otherwise forbidden by structure formation considerations. In other regions of the model parameter space, where the resonance does not appear, another mechanism can operate: a large field suppresses the active-sterile oscillations, but instead sterile neutrinos are produced by the oscillating scalar field when the effective fermion mass crosses zero. In this case, the DM component is cold, and even the $1 \mathrm{keV}$ neutrino is consistent with the cosmic structure formation.
\end{abstract}

DOI: 10.1103/PhysRevD.99.083507

\section{INTRODUCTION}

One of the major problems of present day particle physics is to provide a viable dark matter (DM) candidate, which is an important component of the standard cosmological model. One of the well-motivated candidates is the sterile neutrino. A minimal extension of the Standard Model of particle physics (MSM), providing such a candidate, is Neutrino Minimal Standard Model ( $\nu$ MSM) [1,2], which extends the Standard Model (SM) with three right-handed neutrino partners. If one of the sterile neutrinos $N$ is light, with mass in the $\mathcal{O}(1-100) \mathrm{keV}$ range, it is stable enough on cosmological timescales to form DM [3]. The simplest model is parametrized by sterile neutrino mass $M$ and mixing angle with the active neutrino $\theta_{0}$ [4]. Main constraints on the model arrive from two-body decay $N \rightarrow \nu \gamma$, which leads to an x-ray signal from DM dominated regions of the sky, placing the upper bound on $\theta_{0}$. At

\footnotetext{
*Fedor.Bezrukov@manchester.ac.uk

†chudy@ms2.inr.ac.ru

"gorby@ms2.inr.ac.ru
}

Published by the American Physical Society under the terms of the Creative Commons Attribution 4.0 International license. Further distribution of this work must maintain attribution to the author(s) and the published article's title, journal citation, and DOI. Funded by SCOAP. the same time, production of the correct DM amount $\Omega_{N}=$ $\Omega_{\mathrm{DM}}$ of a sterile neutrino in the early Universe requires some sizeable mixing $\theta_{0}$. The third constraint arrives from the investigation of the cosmic structure formation, which limits the velocity distribution of DM particles. For light DM particles, this results in a significant constraint from below on the mass, which depends on the production spectrum (note that such a light DM can never equilibrate with primordial plasma). Thus, production mechanisms with a cold or mildly warm spectrum are required.

Accommodating all these constraints is a nontrivial task. The simplest mechanism of production in active-sterile oscillations [5] contradicts either x-ray or structure formation constraints. Production in lepton-asymmetric plasma is resonantly enhanced [6] and hence works at a smaller mixing angle, providing a colder spectrum. Still it requires at least $M \gtrsim 5-7 \mathrm{keV}$ [7] and fine-tuning of the model parameters. A number of production mechanisms were proposed to generate the colder neutrino evading the structure formation constraints (see [4] for a review).

In this paper, two new mechanisms of production of the DM sterile neutrino are proposed, both operating in the presence of a coherently oscillating Majoron-type scalar field in the early Universe. Sterile neutrino dynamics of such a system is rather involved. When the effective Majorana mass induced by the scalar background is large, 
no sterile neutrinos are generated from the thermal bath of active neutrinos. At the same time, periodic crossing of zero Majorana mass $M(t)=0$ leads to nontrivial resonance effects both in the active-sterile oscillations (described in the next section) and direct production by the scalar field (effective for a different parameter choice and outlined in the third section). The first mechanism gives the coldest sterile neutrino distribution compared to all other mechanisms relying on active-sterile oscillations and works for small active-sterile mixing angles. The direct production mechanism provides the coldest spectrum for a sterile neutrino produced without relation to active-sterile neutrino mixing.

\section{INDUCED NEUTRINO RESONANCE}

The two main components of the model are the righthanded sterile neutrino $N$ and the light scalar field $\phi$,

$$
\begin{aligned}
\mathcal{L}= & i \bar{\nu} \not \partial+i \bar{N} \not \partial+\frac{1}{2}\left(\partial_{\mu} \phi\right)^{2}+\frac{m_{\phi}^{2}}{2} \phi^{2} \\
& +\left(m_{D} \bar{\nu} N+\frac{M_{0}}{2} \bar{N}^{c} N+\frac{f}{2} \phi \bar{N}^{c} N+\text { H.c. }\right),
\end{aligned}
$$

where $\nu$ is an active SM neutrino, and the Majorana mass is much larger than the Dirac mass, $M_{0} \gg m_{D}$. At present the scalar field is in the vacuum $\phi=0$, and diagonalization of the mass term in (1) yields mixing between heavy (sterile) and light (active) neutrinos,

$$
\theta_{0} \simeq m_{D} / M_{0}
$$

We assume that the scalar is oscillating coherently in the early Universe with amplitude decaying with plasma temperature as $\propto T^{3 / 2}$. This induces the temperature(time-)dependent Majorana mass for $N$

$$
M(t)=M_{0}+M_{A} \sin m_{\phi} t, \quad M_{A} \equiv M_{0}\left(\frac{g_{*} T^{3}}{g_{* e} T_{e}^{3}}\right)^{1 / 2},
$$

where $T_{e}$ is the temperature when the amplitude of oscillating part in (3) equals the bare Majorana mass $M_{A}=M_{0}$. We ignore any difference between the effective numbers of degrees of freedom in plasma, $g_{*}=g_{*}(T)$, which enter energy and entropy densities. Note that the timescale of scalar oscillations $m_{\phi}^{-1}$ is much shorter than that of the change of the amplitude $M_{A}$, which is $2 /(3 H)$ with the Hubble parameter $H$ characterizing the Universe expansion rate.

The coherent evolution of the neutrino states of given momentum $p$ (we also use dimensionless conformal momentum $y \equiv p / T$ when convenient) is described [for relativistic momenta $p \simeq 3 T \gg M(t)$ ] by the equation

$$
i \frac{\partial}{\partial t} \rho=[\mathcal{H}, \rho]-\frac{i}{2}\left\{\Gamma, \rho-\rho_{\mathrm{eq}}\right\}
$$

on the $2 \times 2$ density matrix $\rho$, corresponding to active and sterile neutrinos, with $\rho_{11}\left(\rho_{22}\right)$ being the probability density of the active (sterile) neutrino. Here $\rho_{\text {eq }}=$ $\operatorname{diag}\left(f_{\mathrm{FD}}(y), f_{\mathrm{FD}}(y)\right)$ is the equilibrium Fermi-Dirac (FD) distribution and $\Gamma=\left(\begin{array}{cc}\Gamma_{A} & 0 \\ 0 & 0\end{array}\right)$ is the damping term due to active neutrino interactions in the thermal bath. The Hamiltonian of the system in the plasma reads

$$
\mathcal{H}=\frac{\Delta_{0}}{2}\left(\begin{array}{cc}
-\cos 2 \theta & \sin 2 \theta \\
\sin 2 \theta & \cos 2 \theta
\end{array}\right), \quad \text { where } \Delta_{0}=\frac{\Delta m^{2}}{2 p}
$$

with time-dependent oscillation parameters

$$
\Delta m^{2}=M \sqrt{M^{2}+4 m_{D}^{2}}, \quad \tan \theta=\frac{2 m_{D}}{M+\sqrt{M^{2}+4 m_{D}^{2}}} .
$$

Equation (4) exhibits a nontrivial resonance behavior for neutrino momentum $p_{\text {res }}$ if the typical (properly averaged) frequency of neutrino oscillations is an integer multiple of the frequency of the scalar background oscillations $m_{\phi}$,

$$
\frac{M_{A}^{2}+2 M_{0}^{2}}{4 p_{\text {res }}}=n m_{\phi}, \quad n \in \mathbb{N} .
$$

It can be shown [8] that, in the resonance in the absence of scattering $\Gamma_{A}=0$ (and with odd $n$ ), the $\nu-N$ oscillations proceed effectively (see Fig. 1) with maximum mixing $\rho_{22} \simeq \sin ^{2}\left(\omega_{\text {res }} t / 2\right)$ at frequency

$$
\omega_{\text {res }} \simeq 1.3 m_{\phi} \frac{m_{D}}{M_{A}} n^{1 / 3} \cos \frac{4 M_{0}}{M_{A}} n \simeq 1.3 m_{\phi} \frac{m_{D}}{M_{A}} n^{1 / 3},
$$

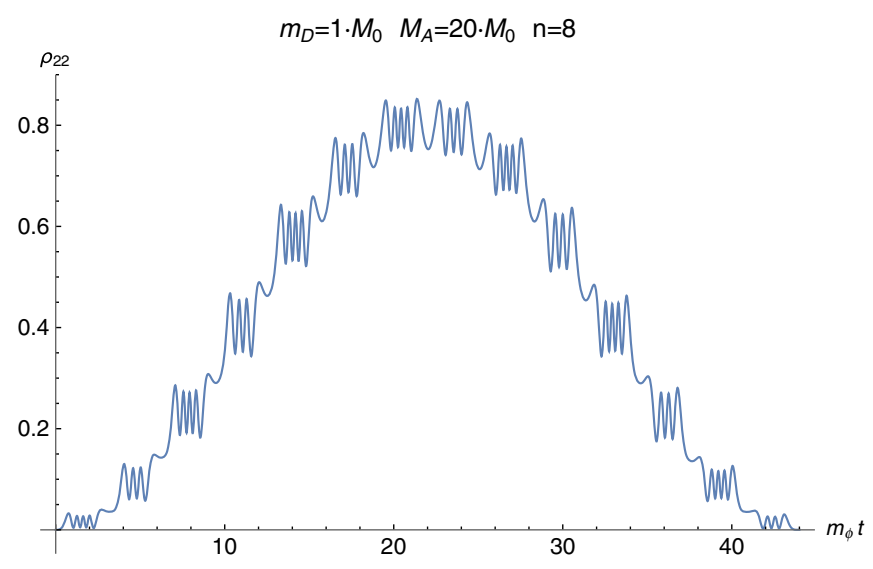

FIG. 1. Example of resonance evolution of transition probability at $n=8$. Relation between $M_{0}$ and $p$ is defined by (5). Fast oscillations with rate $M_{A}^{2} / 4 p \approx 8 m$, slower oscillations with rate $m$ related to the scalar field evolution, and resonant modulation by frequency (6) are shown here. 
which is a good approximation for $M_{A} \gg M_{0}$. Numerically, it works well until $M_{A} \gtrsim 5 M_{0}$.

Note that for our choice of parameters this frequency is much lower than both other typical oscillation frequencies $m_{\phi}$ and $M_{A}^{2} / 4 p$. The resonance is narrow in momentum, with typical width

$$
\frac{\Delta p_{\text {res }}}{p_{\text {res }}} \simeq \frac{\sqrt{2} \omega_{\text {res }}}{n m_{\phi}} \simeq \frac{2}{n} \frac{m_{D}}{M_{A}} \ll 1
$$

We focus at the resonance with the highest momentum, that is $n=1$. Higher resonances are relevant at lower neutrino momentum, while for the purpose of this work the higher momentum region is interesting, because it gives the most important contribution both to the neutrino abundance and to the average neutrino velocity.

For the resonant frequencies, the amount of sterile neutrino reaches Fermi-Dirac distribution after a typical time $\omega_{\text {res }}^{-1}$. However, there are two effects that can prevent the resonance (6) from happening. First, scattering of active neutrino in plasma with $\Gamma_{A}>\omega_{\text {res }}$ slows down the conversion and increases the resonance width [8]. Second, due to the Universe expansion, the conformal momentum $y$ of the resonance changes with temperature and $M_{A}$ (5). At lower temperatures, lower $y$ enter into resonance. However, if the whole resonance band of the width $\Delta y_{\text {res }}=\Delta p_{\text {res }} / T$ (7) moves through a given frequency $y$ faster, than the duration of one oscillation $\omega_{\text {res }}^{-1}$, the resonance becomes ineffective ("narrow"). This happens at high temperatures when

$$
\frac{\Delta y_{\text {res }}}{y_{\text {res }}} \frac{y_{\text {res }}}{\dot{y}_{\text {res }}} \omega_{\text {res }}=\frac{\omega_{\text {res }}^{2}}{\sqrt{2} m_{\phi} H} \lesssim 1,
$$

where $H=T^{2} / M_{\mathrm{Pl}, *}$. Equation (8) defines the temperature above which the resonance is too narrow, and the sterile neutrino distribution function is suppressed, as compared to the Fermi-Dirac distribution.

\section{A. Spectrum with narrow resonance}

The sterile neutrino spectrum $f_{N}$ is given by the FermiDirac distribution for low momenta and is cut off at $y_{s}$ saturating the condition (8). One finds approximately [8]

$$
f_{N}(y)=\frac{f_{\mathrm{FD}}(y)}{\sqrt{1+0.8\left(\frac{y}{y_{s}}\right)^{5}}} .
$$

The spectrum is suppressed at high momenta (see Fig. 2).

Cutoff value $y_{s}$ defines the neutrino abundance today $\Omega_{N} \rho_{\text {crit }}=M_{0} 2 \frac{4}{11} T_{0}^{3} \int 4 \pi y^{2} f_{N}(y) d y$. The DM abundance $\Omega_{N}=\Omega_{\mathrm{DM}}$ is achieved in the mass range $1 \lesssim M_{0} \lesssim$ $20 \mathrm{keV}$ for

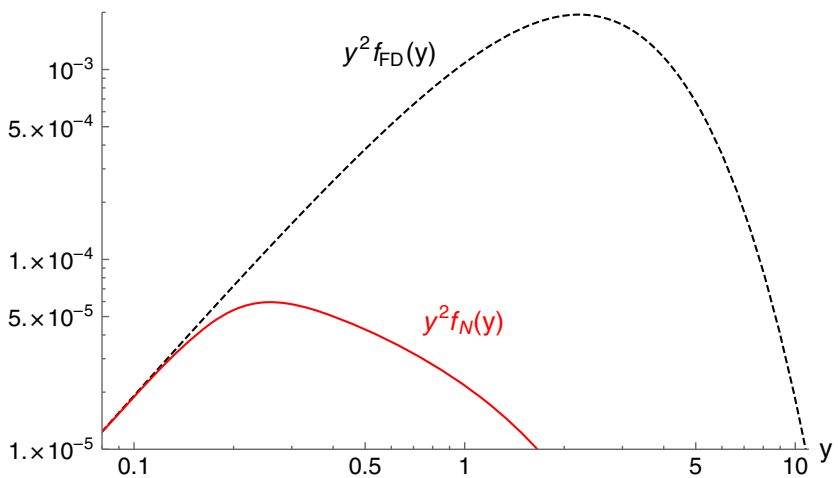

FIG. 2. Fermi-Dirac $f_{\mathrm{FD}}$ and sterile neutrino $f_{N}$ spectra in logarithmic scale.

$$
y_{s} \simeq 0.2\left(\frac{1 \mathrm{keV}}{M_{0}}\right)^{2 / 5} \text {. }
$$

This mode is resonant at the temperature

$T_{s}=3 T_{e}\left(\frac{T_{e}}{11 \mathrm{MeV}}\right)^{1 / 2}\left(\frac{m_{\phi}}{1 \mathrm{eV}}\right)^{1 / 2}\left(\frac{1 \mathrm{keV}}{M_{0}}\right)^{6 / 5}\left(\frac{g_{*, e}}{g_{*, s}}\right)^{1 / 2}$.

The correct DM neutrino abundance is achieved if the resonance becomes too narrow (8) at this temperature, leading to the final result for the mixing angle (8)

$$
\begin{aligned}
\theta_{0} \sim & 3.2 \times 10^{-6}\left(\frac{1 \mathrm{keV}}{M_{0}}\right)^{3}\left(\frac{g_{*, s}}{10.75}\right)^{1 / 4}\left(\frac{m_{\phi}}{1 \mathrm{eV}}\right)^{3 / 4} \\
& \times\left(\frac{T_{e}}{11 \mathrm{MeV}}\right)^{9 / 4} \cdot
\end{aligned}
$$

The dark matter sterile neutrino spectrum (9) is cool with average

$$
\langle\langle y\rangle\rangle=1 / \mathcal{S}^{1 / 3},
$$

where dilution factor $\mathcal{S}=g_{*, s} / 10.75$ accounts for the amount of entropy that was released after sterile neutrino production.

For a conservative choice $\mathcal{S}=1$ (corresponding to the parameter choice in this section) the Ly- $\alpha$ constraint $m_{\mathrm{NRP}}>8 \mathrm{keV}$ [4] is translated in our case to

$$
M_{0}>2.5 \mathrm{keV} .
$$

The main advantage of the proposed generation mechanism is a cold sterile neutrino spectrum (9), which opens a new window for warm dark matter. In what follows we compare in different scenarios average momenta of DM particles calculated just before active neutrino freeze-out, corresponding to $g_{*}=10.75$. Two common mechanisms that rely on the active-sterile neutrino mixing provide a 
warmer spectrum: near thermal distribution for nonresonant production with $\langle\langle y\rangle\rangle_{D W}=3.15$ [5] and resonant production in the presence of lepton asymmetries can lead to average momenta as low as $\left\langle\langle y\rangle_{S F}=1.8 / \mathcal{S}^{1 / 3}\right.$ [9]. There is a wider class of mechanisms that do not rely on activesterile neutrino mixing for the DM generation. The notable examples are decays of thermalized particles leading to $\left\langle\langle y\rangle_{D}=2.45 / \mathcal{S}^{1 / 3}\right.$, where dilution factor $\mathcal{S}$ depends on the production time of sterile neutrinos and is unique for each individual scenario [10,11]. ${ }^{1}$ There are more possibilities if the scalar is extremely weakly coupled and freezes out before decaying to sterile neutrinos, generally leading to a slightly warmer DM $[12,14]$, unless the decay happens at high temperatures [15]. Only the models with a significant entropy release from some beyond SM processes after the sterile neutrino production [16,17] lead to a colder spectrum with $\langle\langle y\rangle\rangle=0.7$.

\section{B. Discussion of the parameter region}

The calculations in the previous subsection are valid if there is a sufficient region of temperatures for the resonance, that is, $T_{s} \gtrsim 3 T_{e}$ (so that $M_{A} \gtrsim 5 M_{0}$ is true). For each value of $\left(M_{0}, \theta\right)$ this requirement limits $T_{e}$ from above (and $m_{\phi}$ from below) [see Fig. 3 and (11) and (12)].

The maximum possible mixing angle is defined by the $x$-ray observational constraints [4]. At low values of the mixing angle, the temperature $T_{e}$ drops below $1 \mathrm{MeV}$, and with active neutrinos out of equilibrium the analysis of (4) is more involved, which defines the lower boundary of the region in Fig. 3.

For large values of $\theta_{0}$, which are close to the nonresonant production line, there is also a contribution from simple active-sterile oscillations at $T<T_{e}$ (cf. [18]), which can be suppressed by lowering $T_{e}$. This leads to additional constraints at low $M_{0}$ and high $\theta_{0}$.

Note that the production in resonantly enhanced oscillations depends on the properties of the scalar sector only via $m_{\phi}$ and $T_{e}$, and not directly on the Yukawa $f$. The Yukawa depends on the initial value of the coherently oscillating scalar field. Thus, it is not required to have large energy density in the scalar field condensate-it only serves to induce the resonance transfer between the active and sterile neutrino sectors. A sufficiently large [but well within perturbativity limits, cf. (17)] value of $f$ makes the mechanism described in the next section inactive for the choice of parameters in Fig. 3. At the same time, for smaller values of $f$ it may be possible to have the scalar contributing to the present DM, leading to multicomponent

\footnotetext{
${ }^{1}$ Frequently, the average momenta at $T \ll 1 \mathrm{MeV}$ are instead given. In this case, decays of thermalized particles at $T \simeq$ $100 \mathrm{GeV}$ lead to $\langle\langle y\rangle\rangle_{D, T \ll 1 \mathrm{MeV}}=0.81$, whereas our result (13) is rescaled as $\langle\langle y\rangle\rangle_{T \ll 1 \mathrm{MeV}}=0.71$. We note that value $g_{*}=$ 3.36 used in $[11,12]$ should be replaced with $g_{*}=43 / 11 \approx 3.9$ (see [13]).
}

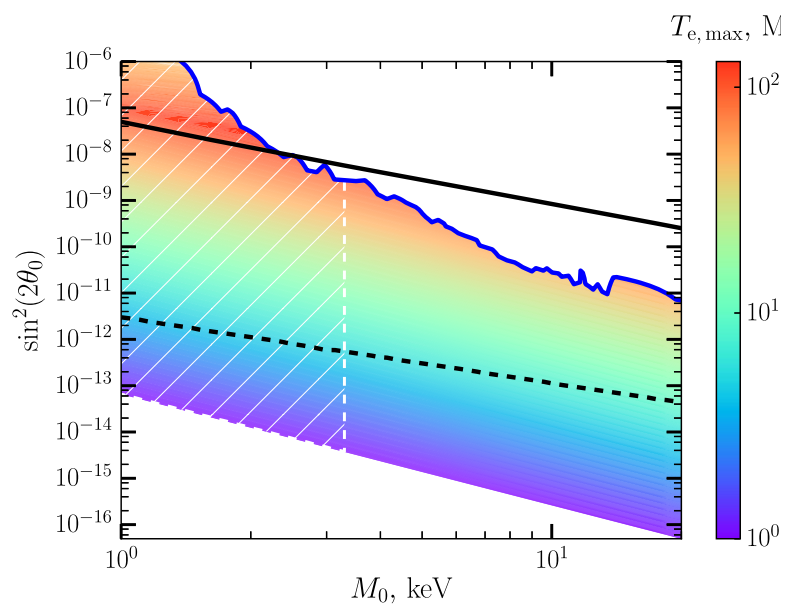

FIG. 3. Parameter scan in the sterile neutrino mass-mixing angle plane (the corresponding scalar masses $10^{-2} \mathrm{eV}<m_{\phi}<$ $M_{0}$ help to avoid additional production of sterile neutrinos from scalar decays at $T \simeq m_{\phi}$ as in Refs. [10-12]). The blue line is the upper limit from x-ray observations [4]. The color region gives the fraction of the sterile neutrino equal to DM $\Omega_{N}=\Omega_{\mathrm{DM}}$. Color indicates the maximal value $T_{e}$ satisfying $T_{e}<T_{s} / 3$ and not leading to overproduction of dark matter via nonresonant generation at $T \lesssim T_{e}$. The white dashed region is excluded by studying the cosmic structure formation. For reference, the black line is for the conventional nonresonant generation mechanism [4], and the black dashed line corresponds to the maximal lepton asymmetry attainable in the $\nu \mathrm{MSM}$ [4].

cold + warm DM with potentially nontrivial structure formation at small scales.

\section{PRODUCTION BY OSCILLATING SCALAR}

In the limit of very low $T_{e}$ (or, alternatively, small $\theta_{0}$ ) neither the mechanism of the last section nor the usual nonresonant neutrino oscillations work [18]. However, for $M_{A}>M_{0}\left(T>T_{e}\right)$, direct production of the sterile neutrino by the scalar field is possible at the moments when the mass $M(t)$ crosses zero $[19,20]$. The process terminates at $T=T_{e}$ (3), and the density of nonrelativistic sterile neutrino produced in this way reads [20] $n_{N} \simeq$ $\left(2 / 6 \pi^{2}\right)\left(M_{0} m_{\phi}\right)^{3 / 2}$. The field amplitude at that moment $\phi_{e}=M_{0} / f$ (1) controls the scalar energy density, $\rho_{\phi}=m_{\phi}^{2} \phi_{e}^{2} / 2$. The scalar field can be stable at cosmological timescales because of kinematics, $m_{\phi}<M_{0}$, and sufficiently small mixing yielding for the scalar width

$$
\frac{\Gamma_{\phi \rightarrow \nu \nu}}{H_{0}} \equiv \theta_{0}^{4} \times \frac{f^{2}}{16 \pi} \frac{m_{\phi}}{H_{0}} \ll 1 .
$$

Then the ratio of energy densities of neutrinos and scalars remain constant, and their present relative contributions are related as 


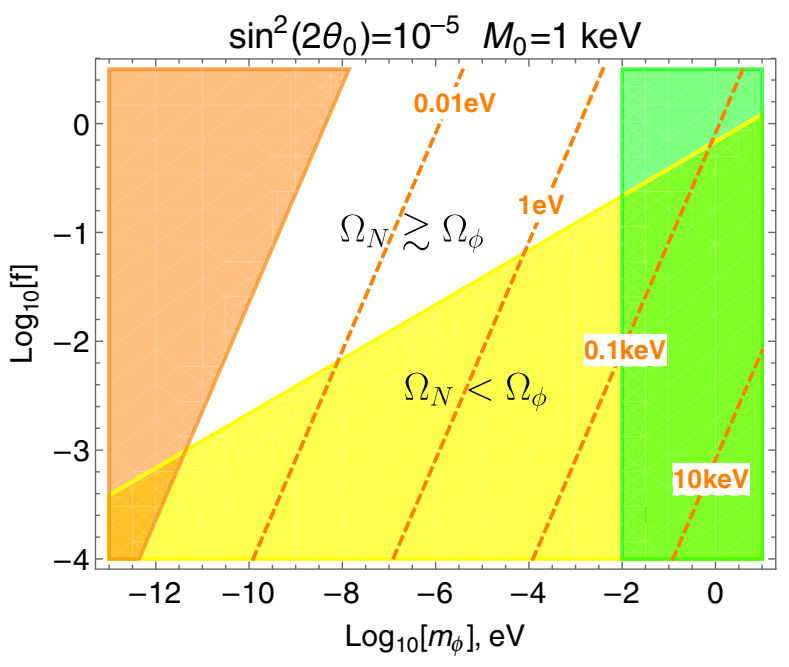

FIG. 4. Direct production of the sterile neutrino by the oscillating scalar field. At each point $\Omega_{\mathrm{DM}}=\Omega_{\phi}+\Omega_{N}$ except for the green region (right) where (15) is violated. Orange region (left) is ruled out by (18). The white region corresponds to the significant contribution of sterile neutrino $\Omega_{N} \gtrsim \Omega_{\phi}$, whereas the yellow field refers to $\Omega_{N}<\Omega_{\phi}$. Dashed lines correspond to several values of $T_{e}$.

$$
\frac{\Omega_{N}}{\Omega_{\phi}}=\frac{2 \rho_{N}\left(T_{e}\right)}{m_{\phi}^{2} \phi_{e}^{2}} \simeq 0.2\left(\frac{f}{0.1}\right)^{2} \sqrt{\frac{M_{0}}{1 \mathrm{keV}}} \sqrt{\frac{0.01 \mathrm{eV}}{m_{\phi}}} .
$$

The total present DM is composed of the mixture of sterile neutrino and the coherently oscillating scalar $\Omega_{\mathrm{DM}}=\Omega_{\phi}+\Omega_{N}$, with the total abundance defined by the scalar field amplitude at high temperatures, which allows us to find the Yukawa coupling

$$
f^{2}=\frac{m_{\phi}^{2} M_{0}^{2}}{2 \Omega_{\mathrm{DM}} \rho_{\text {crit }}} \frac{g_{*, 0} T_{0}^{3}}{g_{* e} T_{e}^{3}} .
$$

To have $N$ mass constant at present we need $T_{e}>T_{0}$, which implies

$$
0.4\left(\frac{0.1}{f}\right)^{\frac{2}{3}}\left(\frac{m_{\phi}}{10^{-10} \mathrm{eV}}\right)^{\frac{2}{3}}\left(\frac{M_{0}}{1 \mathrm{keV}}\right)^{\frac{2}{3}}\left(\frac{g_{*, 0}}{g_{*, e}}\right)^{\frac{1}{3}}>1 .
$$

This production mechanism works at very low $T_{e}$, with DM distributed between the scalar field and sterile neutrino (Fig. 4).

It should be noted that the exact distribution of density between the two types of DM is hard to determine for
$\Omega_{N} / \Omega_{\phi} \gtrsim 1$, as far as the backreaction of the produced sterile neutrino background on the scalar field becomes important. We also stress that neutrino oscillations are efficiently suppressed owing to the choice of very small $T_{e}$ (see Fig. 4). Hence, not only low, but moderate mixing angles are allowed (above usual nonresonant generation limit); the upper limits follow from x-ray searches alone. This option opens new perspectives for direct searches of sterile neutrinos in the future [18].

We emphasize that the sterile neutrino directly produced by the oscillating background is cold. Indeed, at $T=T_{e}$, when modes of the highest momenta $p \lesssim p_{f}$ are produced, all the bigger ones are exponentially suppressed. Since $p_{f} \sim \sqrt{M_{0} m_{\phi}} \ll M_{0}$ [20], the generated sterile neutrinos are nonrelativistic. It allows us to avoid any structure formation constraints.

\section{CONCLUSIONS}

The presence of a coherently oscillating scalar background affects the dynamics of sterile neutrinos significantly. As compared to the traditional sterile neutrino DM scenarios, this allows us either to suppress the creation of sterile neutrinos from active neutrino oscillations in plasma or to enhance these oscillations resonantly. A mechanism converting directly between the scalar background and sterile neutrinos is also possible. In general, combination of all three mechanisms is active at various stages of the thermal history of the Universe, allowing for a rich variety of effects, including generation of warm DM, generation of a sterile neutrino cold DM component along with the scalar DM component, and also cosmologically viable DM sterile neutrinos with relatively large mixing angle. A detailed study of these effects (see also [8]), including more realistic study of the neutrino backreaction on the scalar and quantum corrections to the scalar dynamics, may reveal other interesting scenarios.

\section{ACKNOWLEDGMENTS}

The study of resonant production (A. C. and D. G.) is supported by RSF Grant No. 17-12-01547. The work of F. B. is supported in part by the Lancaster-ManchesterSheffield Consortium for Fundamental Physics, under STFC research Grant No. ST/P000800/1 and by the IPPP associateship. 
[1] T. Asaka, S. Blanchet, and M. Shaposhnikov, Phys. Lett. B 631, 151 (2005).

[2] T. Asaka and M. Shaposhnikov, Phys. Lett. B 620, 17 (2005).

[3] A. D. Dolgov and S. H. Hansen, Astropart. Phys. 16, 339 (2002).

[4] M. Drewes et al., J. Cosmol. Astropart. Phys. 01 (2017) 025.

[5] S. Dodelson and L. M. Widrow, Phys. Rev. Lett. 72, 17 (1994).

[6] X.-D. Shi and G. M. Fuller, Phys. Rev. Lett. 82, 2832 (1999).

[7] J. Baur, N. Palanque-Delabrouille, C. Yeche, A. Boyarsky, O. Ruchayskiy, É. Armengaud, and J. Lesgourgues, J. Cosmol. Astropart. Phys. 12 (2017) 013.

[8] F. Bezrukov, A. Chudaykin, and D. Gorbunov, Scalar induced resonance (to be published).

[9] K. Abazajian, G. M. Fuller, and M. Patel, Phys. Rev. D 64, 023501 (2001).

[10] M. Shaposhnikov and I. Tkachev, Phys. Lett. B 639, 414 (2006).
[11] A. Kusenko, Phys. Rev. Lett. 97, 241301 (2006).

[12] K. Petraki and A. Kusenko, Phys. Rev. D 77, 065014 (2008).

[13] F. Bezrukov and D. Gorbunov, Phys. Rev. D 93, 063502 (2016).

[14] K. Petraki, Phys. Rev. D 77, 105004 (2008).

[15] A. Merle, V. Niro, and D. Schmidt, J. Cosmol. Astropart. Phys. 03 (2014) 028.

[16] F. Bezrukov, H. Hettmansperger, and M. Lindner, Phys. Rev. D 81, 085032 (2010).

[17] A. Kusenko, F. Takahashi, and T. T. Yanagida, Phys. Lett. B 693, 144 (2010).

[18] F. Bezrukov, A. Chudaykin, and D. Gorbunov, J. Cosmol. Astropart. Phys. 06 (2017) 051.

[19] G. F. Giudice, M. Peloso, A. Riotto, and I. Tkachev, J. High Energy Phys. 08 (1999) 014.

[20] D. S. Gorbunov and V. A. Rubakov, Introduction to the Theory of the Early Universe: Cosmological Perturbations and Inflationary Theory (World Scientific, Hackensack, NJ, 2011), p. 489. 